\title{
Coherence Multiplex System Topologies
}

\author{
Arjan Meijerink, Member, IEEE, Robert O. Taniman, Student Member, IEEE, \\ Geert H. L. M. Heideman, and Wim van Etten, Senior Member, IEEE
}

\begin{abstract}
Coherence multiplexing is a potentially inexpensive form of optical code-division multiple access, which is particularly suitable for short-range applications with moderate bandwidth requirements, such as access networks, LANs, or interconnects. Various topologies are known for constructing an optical transmission system in which several channels are coherence-multiplexed in one optical fiber. In this paper, the parallel array, the intrinsic reference ladder (IRL), and the discontinuous series system topologies will be further considered and compared with respect to code orthogonality requirements, theoretical performance, and some practical implementation aspects. A modification to the IRL system is proposed, resulting in a significant improvement in the theoretical performance.
\end{abstract}

Index Terms-Coherence multiplexing (CM), noise analysis, optical code-division multiple access (OCDMA), optical communication, optical network topologies.

\section{INTRODUCTION}

C OHERENCE MULTIPLEXING (CM) is a relatively unknown optical code-division multiplex (OCDM) technique, based on broadband optical sources and strongly unbalanced Mach-Zehnder interferometers (MZIs) [1]-[16]. The channel generation concept in CM is known as coherence modulation, and was first introduced in 1975 by Delisle and Cielo [1]. It is illustrated in Fig. 1, which shows a simplified CM system consisting of one coherence modulator (transmitter), a transmission fiber, and a demodulator (receiver). By means of the MZI, two different versions of the broadband optical source signal $x(t)$ are transmitted, one phase-modulated by a modulating signal $\phi_{\text {mod }}(t)$, and the other unmodulated. Apart from the modulation, these versions have a mutual delay $T_{\mathrm{Tx}}$, due to the path delay difference (PDD) of the MZI. If this delay were much smaller than the coherence time $\tau_{\mathrm{c}}$ of $x(t)$, the two versions would interfere, so that the phase modulation (PM) in the lower branch of the MZI would manifest itself as an intensity modulation (IM) in the output signal $y(t)$ of the MZI. In coherence modulation, however, IM is avoided by choosing $T_{\mathrm{Tx}}$ to be much larger than $\tau_{\mathrm{c}}$, so that the two versions are mutually incoherent,

Manuscript received February 8, 2007; revised July 24, 2007. This work was supported by Philips Research, Eindhoven, The Netherlands, under the Coherence Multiplexing project.

A. Meijerink and W. van Etten are with the Telecommunication Engineering Group, Faculty of Electrical Engineering, Mathematics and Computer Science, University of Twente, $7500 \mathrm{AE}$ Enschede, The Netherlands (e-mail: a.meijerink@ieee.org; etten@ieee.org).

R. O. Taniman is with the Chair for Design and Analysis of Communication Systems, Faculty of Electrical Engineering, Mathematics and Computer Science, University of Twente, 7500 AE Enschede, The Netherlands (e-mail: r.o.taniman@ewi.utwente.nl).

G. H. L. M. Heideman, retired, was with the Telecommunication Engineering Group, Faculty of Electrical Engineering, Mathematics and Computer Science, University of Twente, 7500 AE Enschede, The Netherlands (e-mail: g.h.l.m.heideman@misc.utwente.nl).

Digital Object Identifier 10.1109/JSTQE.2007.905099

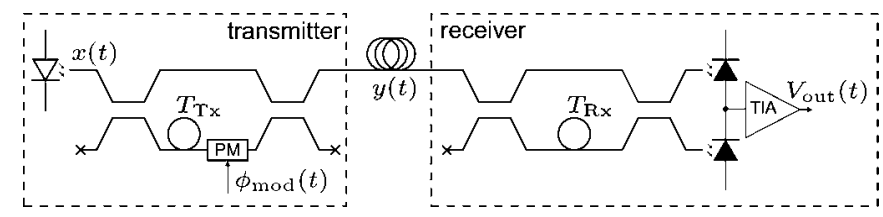

Fig. 1. Simple CM system with one transmitter and one (balanced) receiver (PM: phase modulator, TIA: transimpedance amplifier).

no interference occurs, and $\phi_{\text {mod }}(t)$ is not visible in the intensity of $y(t)$. Demodulation is performed by means of another MZI, with a PDD $T_{\mathrm{Rx}}$. It will split the two versions into four versions. It can be verified that two of these have a mutual delay difference that equals $T_{\mathrm{Rx}}-T_{\mathrm{Tx}}$, so if $T_{\mathrm{Rx}}$ is equal to $T_{\mathrm{Tx}}$, the corresponding optical signal terms are mutually coherent, resulting in interference. Since only one of these two terms is modulated, this results in the desired PM-IM conversion. All the other terms are mutually incoherent and result in optical beat interference (OBI) noise. It can be verified that no PM-IM conversion occurs when $T_{\mathrm{Rx}}-T_{\mathrm{Tx}}$ is much larger than $\tau_{\mathrm{c}}$, so the relation between the PDDs in transmitter and receiver determines whether the corresponding coherence-modulated channel is demodulated or not. Optical detection is assumed to be performed by a balanced photodiode pair, as it can be shown to result in a superior OBI noise performance compared to direct detection [9], [10].

Cielo and Delisle also proposed how several coherencemodulated channels can be multiplexed in a common optical transmission fiber, by cascading multiple coherence modulators and using a common optical source [2]. Some alternatives were proposed by Brooks et al. [3], and analyzed by Wentworth [6], for application in an optical interferometric sensor system. Apart from the serial configuration, which they termed the discontinuous series (DS) system, they proposed the continuous series (CS) system, the extrinsic reference ladder (ERL) system, and the intrinsic reference ladder (IRL) system. The IRL system is basically a parallel array (PA) of coherence modulators that are illuminated by a common optical source. A more straightforward (and also more flexible and power effective) approach, is to build a PA in which each coherence modulator is illuminated by its own optical source, as proposed by Goedgebuer and Hamel [5]. A considerable performance improvement can be achieved when the MZIs in the IRL system are integrated to one MZI with a common reference branch, and several modulated paths with different PDDs with respect to the reference branch [13], [15]. This is termed the single IRL (SIRL) system.

$\mathrm{CM}$ has several properties in common with other OCDM techniques, such as simultaneous (asynchronous) operation in a common wavelength band, low access delay, gradual performance degradation with increasing number of channels (the so-called soft capacity), and encoding and decoding in the 
optical domain. Specifically, CM can be categorized as a coherent OCDM technique, as detection is based on coherent matched filtering [9]. The "optical code" in CM can be defined in different ways.

1) The broadband optical source signal $x(t)$ can be considered as a truly random scrambling code. The scrambling code is, in this case, transmitted along with the scrambled data signal, because the code is unknown at the receiver.

2) The $\cos ^{2}(\cdot)$-shaped power transfer function of the transmitter's MZI can be considered as a periodic spectral code, because the MZI more or less alters the spectrum of the broadband optical source signal $x(t)$. The period of the transfer function is equal to $1 / T_{\mathrm{Tx}}$, so the spectral codes of different transmitters are orthogonal within the source spectrum if the values of the PDDs are sufficiently different. We will elaborate more on this in Sections II-D, III-D, and IV-D.

The particular advantages of CM compared to many other known OCDM techniques-such as pulse time encoding, spectral amplitude and/or phase encoding, and wavelength hopping-are as follows.

1) $\mathrm{CM}$ does not require short pulses and/or time-gating: the data signal is directly modulated on a (broadband) continuous-wave optical carrier, and is simply extracted from the detected optical signal by electrical filtering. This relaxes the bandwidth requirements of the optical modulators and detectors, and makes the CM system transparent to the transmitted data signal.

2) Simple optical sources such as LEDs or superluminescent LEDs (SLEDs) can be used as broadband optical sources. These do not need to be stabilized and/or tunable in center wavelength.

3) Relatively simple optical circuits are required for performing the encoding and decoding, namely unbalanced MZIs, which can be easily integrated as planar optical waveguide circuits.

4) The concept is relatively robust in the sense that environmental changes (such as temperature drift) and/or aging do not result in crosstalk between channels.

$\mathrm{CM}$ also has particular disadvantages, some of which are common to other OCDM techniques.

1) The quality of the detected signal degrades with increasing number of active channels, in this case, due to the OBI noise.

2) The large spectral width of the optical carrier signal makes the modulated optical signal vulnerable to chromatic dispersion in the transmission fiber.

3) The transmitted optical power is shared by all receivers.

4) The interfering optical signals at the decoders have to be matched in phase and polarization.

The OBI noise, chromatic dispersion, and low power budget fundamentally limit the number of $\mathrm{CM}$ channels that can be multiplexed, the data rates that can be accommodated, and the distances that can be spanned. Phase and polarization matching will not be an issue when the optical encoders and decoders are integrated as planar optical waveguide circuits. Also considering the advantages mentioned before, this should result in a relatively inexpensive implementation of the technique. $\mathrm{CM}$, therefore, seems to be suitable for relatively short-range applications with moderate number of channels and data rates, with particular focus on low costs rather than on network capacity. Examples of such applications are optical subscriber networks, local area networks, and interconnects. Moreover, the transparency of the transmission concept enables $\mathrm{CM}$ to be used for analog transmission, for example, optical RF feeding [17].

In this paper, the different system topologies for implementing CM will be considered. The CS and ERL system will not be included in this study, however, as they require two separate transmission paths between each transmitter and receiver. Although this might be practical in an optical sensor application (typically with short fiber length), it is not desirable in an optical fiber communication system, because the polarization states of the optical signals at the outputs of the two optical fibers need to be matched in order to achieve optimal interference. Moreover, the differential length mismatch between the two fibers would have significant impact on the performance of the system [8]. Therefore, only the remaining three topologies will be considered.

The paper will be organized as follows. The PA, SIRL system, and DS system will be considered in further detail in Sections II, III, and IV, respectively, with particular focus on design constraints and theoretical performance. In Section V, they will be compared with respect to some practical aspects. The paper ends with conclusions in Section VI.

\section{The PARALlel ARRAY}

\section{A. System Description}

The PA is the most straightforward way of multiplexing coherence-modulated channels, and is shown in Fig. 2. It consists of $N$ coherence modulators and $N$ (balanced) coherence demodulators. Each coherence modulator $i$ is illuminated by a separate optical source signal, which will be described by the normalized scalar wave representation of the optical field [15], [18], with (complex) preenvelope $x_{i}(t)$. The random behavior of $x_{i}(t)$ will be described by the thermal light model, which implies that $x_{i}(t)$ is considered as a circular complex Gaussian bandpass process [6], [14], [15], [18]. In the particular case of LEDs or superluminescent diodes, the spectrum is approximately Gaussian, so that the autocorrelation function of $x_{i}(t)$ is

$$
\begin{aligned}
R_{x^{*} x}(\tau) & \triangleq E\left[x_{i}^{*}(t) x_{i}(t+\tau)\right] \\
& =2 P_{\text {in }} \exp \left(-\pi\left(\tau / \tau_{\mathrm{c}}\right)^{2} / 2\right) \exp \left(j 2 \pi f_{\mathrm{c}} \tau\right)
\end{aligned}
$$

where $P_{\text {in }}$ is the average optical power, $f_{\mathrm{c}}$ is the optical center frequency, and $\tau_{\mathrm{c}}$ is the coherence time, which is defined as

$$
\tau_{\mathrm{c}} \triangleq \int\left|R_{x^{*} x}(\tau)\right|^{2} \mathrm{~d} \tau / R_{x^{*} x}^{2}(0) \text {. }
$$

Each MZI has a PDD $T_{\mathrm{Tx}, i}$ that is much larger than $\tau_{\mathrm{c}}$, and the phase-modulating signals are denoted as $\phi_{\bmod , i}(t)$. The couplers in the MZIs are assumed to be uniform, and the excess losses of the MZIs are denoted by $L_{\mathrm{Tx}}$. 


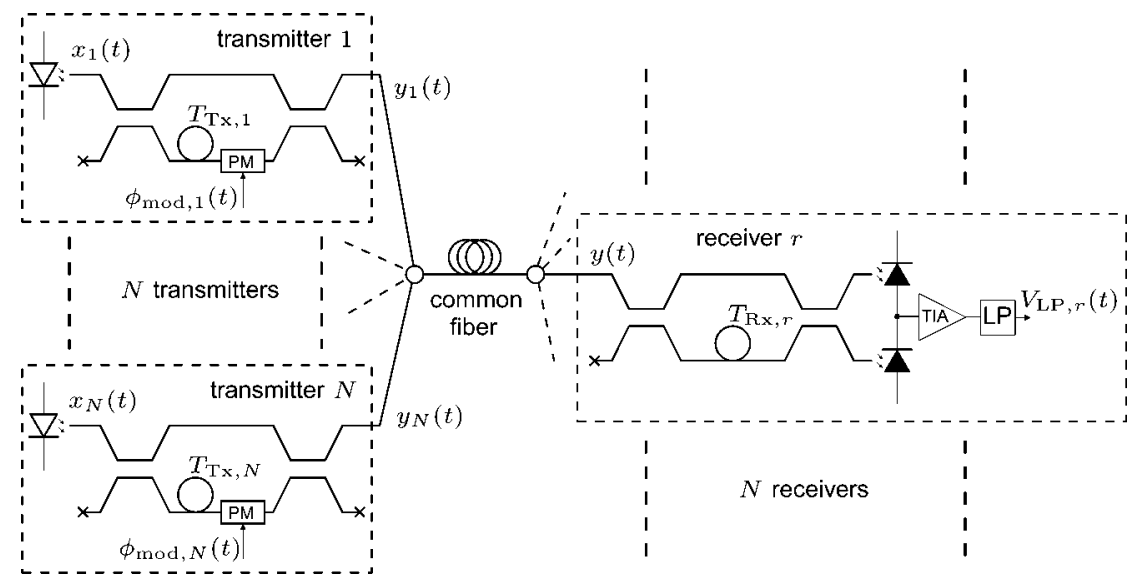

Fig. 2. PA of $N$ coherence modulators and demodulators.

The output signals $y_{i}(t)$ of the coherence modulators are combined into the common transmission fiber using an optical combiner. The aggregate signal is then distributed over the $N$ receivers by means of an optical splitter. When the difference in propagation delay is ignored and splitters and combiners are assumed to be uniform, this implies that each coherence demodulator receives an identical signal $y(t)$. The optical losses in the network-due to intrinsic combining and splitting losses, excess losses, coupling losses, and propagation losses-are denoted by $L_{\mathrm{nw}}$. Depending on the fiber length, chromatic dispersion in the transmission fibers might result in a significant performance penalty [12], [15]. However, chromatic dispersion will be ignored in this paper, as the focus will be on the topology differences, whereas the dispersion penalty can be shown to be the same for each topology.

Each coherence demodulator has an MZI with uniform couplers, PDD $T_{\mathrm{Rx}, r} \gg \tau_{\mathrm{c}}$, and excess loss $L_{\mathrm{Rx}}$. The outputs of the MZI are detected by a balanced receiver, consisting of linear photodiodes with identical responsivities $R_{\mathrm{pd}}$. The photonelectron conversion process is modeled as an inhomogeneous doubly stochastic Poisson process [14], [15]. The output current is amplified by a transimpedance amplifier (TIA) with transimpedance $Z_{\text {TIA }}$ and equivalent input noise current with power spectral density $S_{\mathrm{th}}$. Its output voltage is filtered by means of a low-pass filter with impulse response $h_{\mathrm{LP}}(t)$, resulting in an output voltage $V_{\mathrm{LP}, r}(t)$.

\section{B. Multiplexed Signal}

When the combined signals are assumed to have matched polarization states, $y(t)$ can be written as

$$
\begin{aligned}
y(t)= & \frac{1}{\sqrt{L_{\mathrm{nw}}}} \sum_{i=1}^{N} y_{i}(t)=\frac{1}{2 \sqrt{L_{\mathrm{Tx}} L_{\mathrm{nw}}}} \\
& \times \sum_{i=1}^{N}\left[x_{i}(t)-x_{i}\left(t-T_{\mathrm{Tx}, i}\right) \exp \left(j \phi_{\mathrm{mod}, i}(t)\right)\right] .
\end{aligned}
$$

When the modulating signals $\phi_{\bmod , i}(t)$ are considered as deterministic signals with bandwidths that are much smaller than $1 / \tau_{\mathrm{c}}$ and the inverse of the PDDs, the autocorrelation function of the (nonstationary) signal $y(t)$ can be written as

$$
\begin{aligned}
R_{y^{*} y}\left(t_{1}, t_{2}\right) \triangleq & E\left[y^{*}\left(t_{1}\right) y\left(t_{2}\right)\right] \\
\approx & \frac{1}{4 L_{\mathrm{Tx}} L_{\mathrm{nw}}} \sum_{i=1}^{N}\left[2 R_{x^{*} x}\left(t_{2}-t_{1}\right)\right. \\
& -R_{x^{*} x}\left(t_{2}-t_{1}-T_{\mathrm{Tx}, i}\right) \exp \left(j \phi_{\bmod , i}\left(t_{1}\right)\right) \\
& \left.-R_{x^{*} x}\left(t_{2}-t_{1}+T_{\mathrm{Tx}, i}\right) \exp \left(-j \phi_{\mathrm{mod}, i}\left(t_{1}\right)\right)\right]
\end{aligned}
$$

where it is assumed that all source signals $x_{i}(t)$ 's are independent and have the same autocorrelation function $R_{x^{*} x}(\tau)$.

\section{Receiver Output Signal}

It can be proven [14], [15] that the expected value of the output voltage of the low-pass filter is related to the autocorrelation function of $y(t)$ as

$$
\begin{aligned}
E\left[V_{\mathrm{LP}, r}(t)\right]= & -\frac{Z_{\mathrm{TIA}} R_{\mathrm{pd}}}{2 L_{\mathrm{Rx}}} \int h_{\mathrm{LP}}(t-\rho) \\
& \times \operatorname{Re}\left\{R_{y^{*} y}\left(\rho-T_{\mathrm{Rx}}, \rho\right)\right\} \mathrm{d} \rho
\end{aligned}
$$

where $\operatorname{Re}\{\cdot\}$ denotes the real part. Substituting (4) and taking into account that $R_{x^{*} x}(\tau)$ is negligible for $|\tau| \gg \tau_{\mathrm{c}}$, this can be written as

$$
\begin{aligned}
& E\left[V_{\mathrm{LP}, r}(t)\right] \approx \frac{Z_{\mathrm{TIA}} R_{\mathrm{pd}}}{8 L_{\mathrm{Tx}} L_{\mathrm{nw}} L_{\mathrm{Rx}}} \int h_{\mathrm{LP}}(t-\rho) \\
& \quad \times \sum_{i=1}^{N} \operatorname{Re}\left\{R_{x^{*} x}\left(T_{\mathrm{Rx}, r}-T_{\mathrm{Tx}, i}\right) \exp \left(j \phi_{\mathrm{mod}, i}(\rho)\right) \mathrm{d} \rho\right\} .
\end{aligned}
$$

Hence, it follows that the output signal of each receiver $r$ depends only on the modulating signal of the corresponding transmitter $r$ if $\left|T_{\mathrm{Rx}, r}-T_{\mathrm{Tx}, i}\right| \gg \tau_{\mathrm{c}}$ for $i \neq r$. In other words, the transmitters' spectral codes (mentioned in Section I) can be considered orthogonal in that case. The maximum amplitude is obtained when $\left|T_{\mathrm{Rx}, r}-T_{\mathrm{Tx}, r}\right| \ll \tau_{\mathrm{c}}$, resulting in

$$
\begin{aligned}
E\left[V_{\mathrm{LP}, r}(t)\right] \approx & \frac{Z_{\mathrm{TIA}} R_{\mathrm{pd}} P_{\mathrm{in}}}{4 L_{\mathrm{Tx}} L_{\mathrm{nw}} L_{\mathrm{Rx}}} \int h_{\mathrm{LP}}(t-\rho) \\
& \times \cos \left(\Delta \phi_{r}+\phi_{\mathrm{mod}, r}(\rho)\right) \mathrm{d} \rho
\end{aligned}
$$




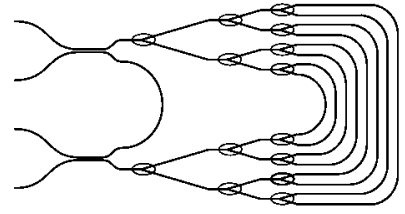

(a)

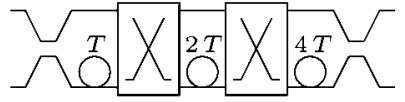

(b)
Fig. 3. Two examples of MZIs with adjustable PDDs. (a) Binary tree configuration of parallel delay lines and Y-switches. (b) Series concatenation with cross-bar switches.

where $\Delta \phi_{r} \triangleq 2 \pi f_{\mathrm{c}}\left(T_{\mathrm{Rx}, r}-T_{\mathrm{Tx}, r}\right)$ is the phase offset between the interfering optical signals due to small differences between the PDDs in transmitter and receiver $r$. In case of digital transmission using bipolar phase-shift keying (BPSK) modulation $\left[\phi_{\bmod , r}(t)=0\right.$ for a binary zero and $\phi_{\bmod , r}(t)=\pi$ for a binary one], the amplitude of the output signal is proportional to $\cos \left(\Delta \phi_{r}\right)$, and hence, is maximized by setting $\Delta \phi_{r}=0$. This can be achieved by means of a phase synchronization scheme using a feedback loop and a frequency dithering technique [19]. Alternatively, the need for phase synchronization can be circumvented by applying self-heterodyning instead of self-homodyning, using an optical frequency shifter [8], or by applying a phase diversity scheme [14], [15]. This will not be considered in further detail here. In this paper, it will, from now on, simply be assumed that the optical phases are somehow synchronized, so it is assumed that $\Delta \phi_{r}=0$.

\section{Choice of the Path Delay Differences}

From the previous section, it follows that the transmitters' PDDs $T_{\mathrm{Tx}, i}$ should be chosen such that each individual value and their mutual differences are all much larger than $\tau_{\mathrm{c}}$, in order to achieve orthogonality of the transmitters' spectral codes. A straightforward choice is to choose some delay value $T$ that is much larger than $\tau_{\mathrm{c}}$, and choose the PDDs to be integer multiples of $T$, so $T_{\mathrm{Tx}, i}=i T$. Using (1), (4), and (5), it can actually be verified [15] that when there are $N=16$ transmitters, and $T \geq 3 \tau_{\mathrm{c}}$, the theoretical crosstalk due to residual coherence is already below $-90 \mathrm{~dB}$.

As an example, when we consider a PA using light sources with $\tau_{\mathrm{c}}=0.1 \mathrm{ps}$, and integrated MZIs with a waveguide group index in the order of 1.5 , the smallest path length difference (PLD) will be $3 \times 10^{8} \times 3 \times 0.1 \times 10^{-12} / 1.5 \mathrm{~m}=60 \mu \mathrm{m}$, and the largest PLD will be $16 \times 60 \mu \mathrm{m} \approx 1 \mathrm{~mm}$. MZIs with such PLD values can be easily fabricated in integrated optics technology.

In some applications, it might be desirable to have adjustable PDD values in the transmitter and/or receiver, so that a transmitter can address a particular receiver and/or a receiver can tune to a particular transmitter. This can be done by means of multiple delays and switches. Two examples are shown in Fig. 3. In the MZI in Fig. 3(a), one of the interferometer arms is replaced by a binary tree configuration of parallel delay lines, from which one delay line can be selected by properly setting the Y-switches. The MZI in Fig. 3(b) consists of multiple stages that are connected by cross-bar switches. The PDD in each stage is twice the
PDD of the preceding stage. When a switch is in the bar state, the PDD of the two adjacent stages are effectively summed, whereas they are effectively subtracted when the switch is in the cross-state.

An MZI can also be alternately used for modulation and demodulation in the same channel, so that a half-duplex link can be established [11].

\section{E. Noise Analysis}

The output signal of the receiver is assumed to be mainly corrupted by three types of noise [6], [7], [11], [14], [15].

1) Source-induced noise: It is caused by the random behavior of the optical source signal, resulting in random fluctuations in the power of the detected signal. Source intensity noise results in random fluctuations of the desired interference term, and source phase noise results into OBI noise, as explained in Section I.

2) Shot noise: This occurs due to the random arrival times of photons.

3) Thermal receiver noise: This is caused by the random motion of electrons and holes in the electronic components.

Following a similar procedure as in [14] and [15], the noise variance at the output of the low-pass filter in receiver $r$ can be shown to be related to the autocorrelation of the received signal $y(t)$ as

$$
\begin{aligned}
\sigma_{V_{\mathrm{LP}, r}(t)}^{2} \triangleq E\left[V_{\mathrm{LP}, r}^{2}(t)\right]-E^{2}\left[V_{\mathrm{LP}, r}(t)\right] \\
=\frac{Z_{\mathrm{TIA}}^{2} R_{\mathrm{pd}}^{2}}{8 L_{\mathrm{Rx}}^{2}} \iint h_{\mathrm{LP}}\left(t-\rho_{1}\right) h_{\mathrm{LP}}\left(t-\rho_{2}\right) \\
\quad \times \operatorname{Re}\left\{R_{y^{*} y}\left(\rho_{1}, \rho_{2}\right) R_{y^{*} y}\left(\rho_{2}-T_{\mathrm{Rx}, r}, \rho_{1}-T_{\mathrm{Rx}, r}\right)\right. \\
\left.\quad+R_{y^{*} y}\left(\rho_{1}-T_{\mathrm{Rx}, r}, \rho_{2}\right) R_{y^{*} y}\left(\rho_{2}-T_{\mathrm{Rx}, r}, \rho_{1}\right)\right\} \mathrm{d} \rho_{1} \mathrm{~d} \rho_{2} \\
+\frac{Z_{\mathrm{TIA}}^{2} R_{\mathrm{pd}} e}{4 L_{\mathrm{Rx}}} \int h_{\mathrm{LP}}^{2}(t-\rho)\left[R_{y^{*} y}(\rho, \rho)\right. \\
\left.\quad+R_{y^{*} y}\left(\rho-T_{\mathrm{Rx}, r}, \rho-T_{\mathrm{Rx}, r}\right)\right] \mathrm{d} \rho \\
+Z_{\mathrm{TIA}}^{2} S_{\mathrm{th}} \int h_{\mathrm{LP}}^{2}(\rho) \mathrm{d} \rho
\end{aligned}
$$

where $e$ is the charge of an electron $\left(1.60 \times 10^{-19} \mathrm{C}\right)$. The three terms correspond to source-induced noise, shot noise, and thermal noise, respectively. Using (4), taking into account that $R_{x^{*} x}(\tau)$ is negligible for $|\tau| \gg \tau_{\mathrm{c}}$, and applying phase averaging [6], [15], one can find for the PA

$$
\begin{gathered}
R_{y^{*} y}\left(\rho_{1}, \rho_{2}\right) R_{y^{*} y}\left(\rho_{2}-T_{\mathrm{Rx}, r}, \rho_{1}-T_{\mathrm{Rx}, r}\right) \\
=\frac{1}{16 L_{\mathrm{Tx}}^{2} L_{\mathrm{nw}}^{2}}\left\{4 N^{2}\left|R_{x^{*} x}\left(\rho_{2}-\rho_{1}\right)\right|^{2}\right. \\
+\sum_{i=1}^{N}\left[\left|R_{x^{*} x}\left(\rho_{2}-\rho_{1}-T_{\mathrm{Tx}, i}\right)\right|^{2}\right. \\
\left.\left.\quad+\left|R_{x^{*} x}\left(\rho_{2}-\rho_{1}+T_{\mathrm{Tx}, i}\right)\right|^{2}\right]\right\}
\end{gathered}
$$




$$
\begin{aligned}
& R_{y^{*} y}\left(\rho_{1}-T_{\mathrm{Rx}, r}, \rho_{2}\right) R_{y^{*} y}\left(\rho_{2}-T_{\mathrm{Rx}, r}, \rho_{1}\right) \\
& \quad=\frac{1}{16 L_{\mathrm{Tx}}^{2} L_{\mathrm{nw}}^{2}}\left|R_{x^{*} x}\left(\rho_{2}-\rho_{1}\right)\right|^{2} \exp \left(j 2 \phi_{\mathrm{mod}, r}\left(\rho_{1}\right)\right) \\
& R_{y^{*} y}(\rho, \rho)=R_{y^{*} y}\left(\rho-T_{\mathrm{Rx}, r}, \rho-T_{\mathrm{Rx}, r}\right)=\frac{P_{\mathrm{in}} N}{L_{\mathrm{Tx}} L_{\mathrm{nw}}} .
\end{aligned}
$$

Now assume that the CM system is used for digital transmission using BPSK modulation, so $\phi_{\bmod , r}(t)$ is a rectangular data signal taking symbol values 0 and $\pi$ with pulse duration $T_{\mathrm{s}}$. Also assume that $h_{\mathrm{LP}}(t)$ is matched to these pulses, so the low-pass filter is, in fact, an integrate-and-dump filter with integration time $T_{\mathrm{s}}$. The expected output signal of the low-pass filter at the sampling instants then follows from (7) as

$$
E\left[V_{\mathrm{LP}, r}(t)\right] \approx \pm \frac{Z_{\mathrm{TIA}} R_{\mathrm{pd}} P_{\mathrm{in}} T_{\mathrm{s}}}{4 L_{\mathrm{Tx}} L_{\mathrm{nw}} L_{\mathrm{Rx}}}
$$

Substituting (9) through (11) in (8), assuming that $T_{\mathrm{S}} \gg T_{\mathrm{Tx}, i}$, and using (2), it can be shown that

$$
\begin{aligned}
\sigma_{V_{\mathrm{LP}, r}(t)}^{2} \approx & Z_{\mathrm{TIA}}^{2} T_{\mathrm{s}}\left[\frac{\left(4 N^{2}+2 N+1\right) R_{\mathrm{pd}}^{2} P_{\mathrm{in}}^{2} \tau_{\mathrm{c}}}{32 L_{\mathrm{Tx}}^{2} L_{\mathrm{Rx}}^{2} L_{\mathrm{nw}}^{2}}\right. \\
& \left.+\frac{N R_{\mathrm{pd}} P_{\mathrm{in}} e}{2 L_{\mathrm{Tx}} L_{\mathrm{Rx}} L_{\mathrm{nw}}}+S_{\mathrm{th}}\right] .
\end{aligned}
$$

The SNR can, hence, be written as

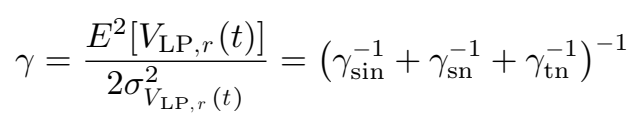

where

$$
\begin{gathered}
\gamma_{\mathrm{sin}}=\frac{T_{\mathrm{s}}}{\left(4 N^{2}+2 N+1\right) \tau_{\mathrm{c}}} \\
\gamma_{\mathrm{sn}}=\frac{R_{\mathrm{pd}} P_{\mathrm{in}} T_{\mathrm{s}}}{16 N L_{\mathrm{Tx}} L_{\mathrm{Rx}} L_{\mathrm{nw}} e} \\
\gamma_{\mathrm{tn}}=\frac{R_{\mathrm{pd}}^{2} P_{\mathrm{in}}^{2}}{32 L_{\mathrm{Tx}}^{2} L_{\mathrm{Rx}}^{2} L_{\mathrm{nw}}^{2} S_{\mathrm{th}}}
\end{gathered}
$$

are the signal-to-source-induced-noise ratio, signal-to-shotnoise ratio, and signal-to-thermal-noise ratio, respectively. Since $T_{\mathrm{S}} \gg \tau_{\mathrm{c}}$, it can be assumed that the integration time of the lowpass filter is much larger than the correlation time of the sourceinduced noise, so that the central limit theorem applies, and the source-induced noise at the output of the filter can be considered as Gaussian distributed. In case of large received powers, the shot noise can also be considered as Gaussian distributed. And finally, the thermal noise can also be considered as Gaussian noise. Therefore, the bit error rate (BER) can be calculated using the classical result for polar nonreturn-to-zero signalling in additive white Gaussian noise [20], resulting in

$$
P_{\mathrm{e}}=Q(\sqrt{2 \gamma})
$$

where the Gaussian tail probability $Q(\cdot)$ is defined as

$$
Q(x) \triangleq \frac{1}{\sqrt{2 \pi}} \int_{x}^{\infty} \exp \left(\frac{-z^{2}}{2}\right) \mathrm{d} z
$$

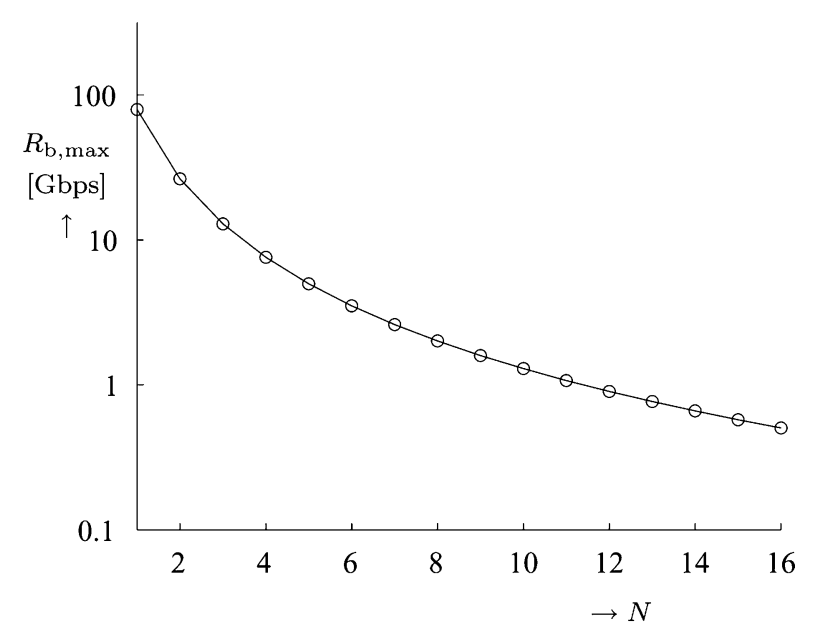

(a)

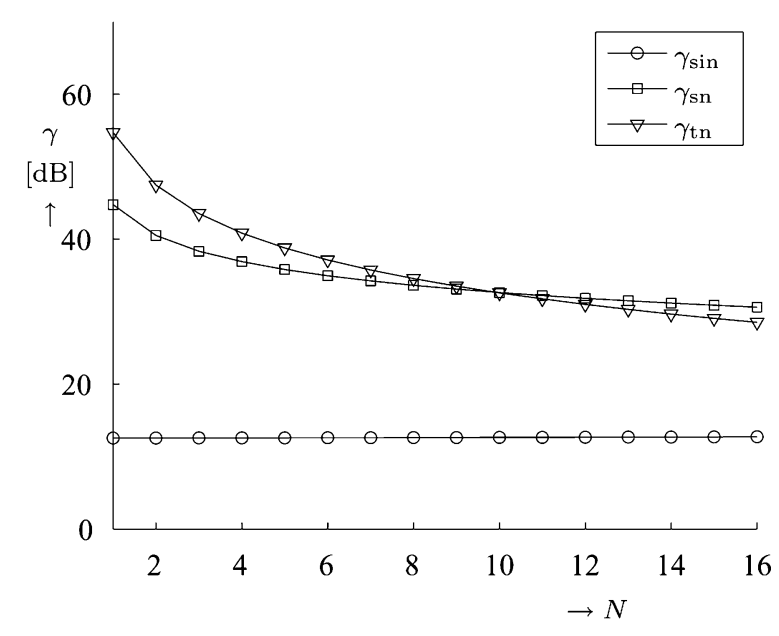

(b)

Fig. 4. (a) Maximum bit rate per channel $R_{\mathrm{b}, \max }$ as a function of number of channels $N$ in a PA with BPSK modulation. (b) Corresponding SNRs.

\section{F. Numerical Example}

As an example, consider the case in which the optical sources couple $P_{\text {in }}=10 \mathrm{~mW}$ of optical power into each MZI, and have a coherence time $\tau_{\mathrm{c}}=0.1 \mathrm{ps}$. The splitters and combiners have an intrinsic splitting loss $N$, and are assumed to have an excess loss of $0.1 \mathrm{~dB}$. Hence, when propagation losses in the transmission fiber are neglected, the total network losses can be calculated to be $L_{\mathrm{nw}} \approx 1.05 N^{2}$. When the MZIs in transmitters and receivers are assumed to have an excess loss of $0.5 \mathrm{~dB}$, we have $L_{\mathrm{Tx}}=L_{\mathrm{Rx}} \approx 1.12$. The photodiodes in the optical receivers are assumed to have a responsivity $R_{\mathrm{pd}} \approx 0.8 \mathrm{~A} / \mathrm{W}$, and the TIAs are assumed to have an rms-equivalent input current in the order of $7 \mathrm{pA} / \sqrt{\mathrm{Hz}}$, so we have $S_{\mathrm{th}} \approx 5 \times 10^{-23} \mathrm{~A}^{2} / \mathrm{Hz}$.

From (18), it follows that a maximum BER of $10^{-9}$ requires a minimum SNR $\gamma=18$ (12.6 dB). Using (14) through (17), the maximum bit rate per channel $R_{\mathrm{b}}=1 / T_{\mathrm{s}}$ that can be achieved with a BER of $10^{-9}$ can now be calculated as a function of the number of channels $N$, and is plotted in Fig. 4(a). The corresponding SNRs are shown in Fig. $4 \mathrm{~b}$.

From Fig. 4(a), it follows that at most 3 channels can be supported at $10 \mathrm{~Gb} / \mathrm{s}$, and at most 11 channels can be supported 


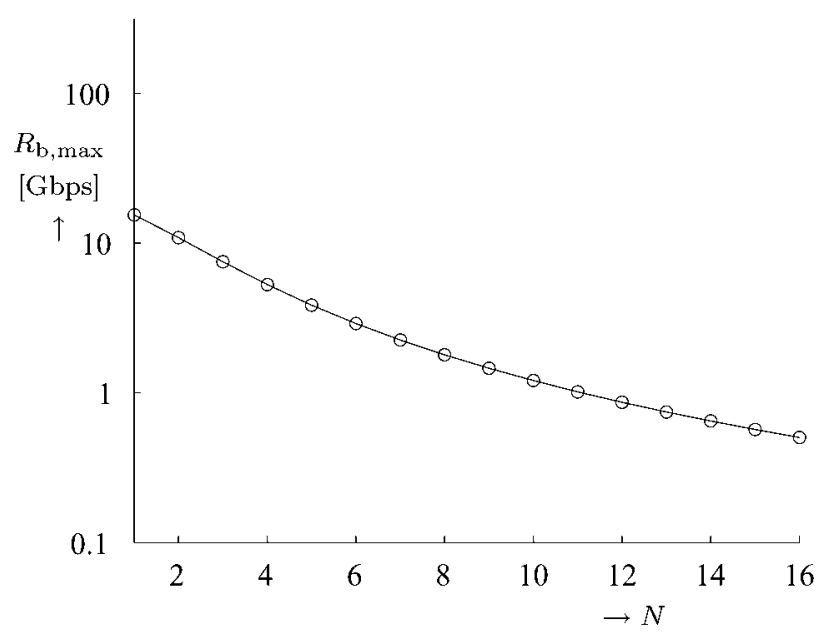

(a)

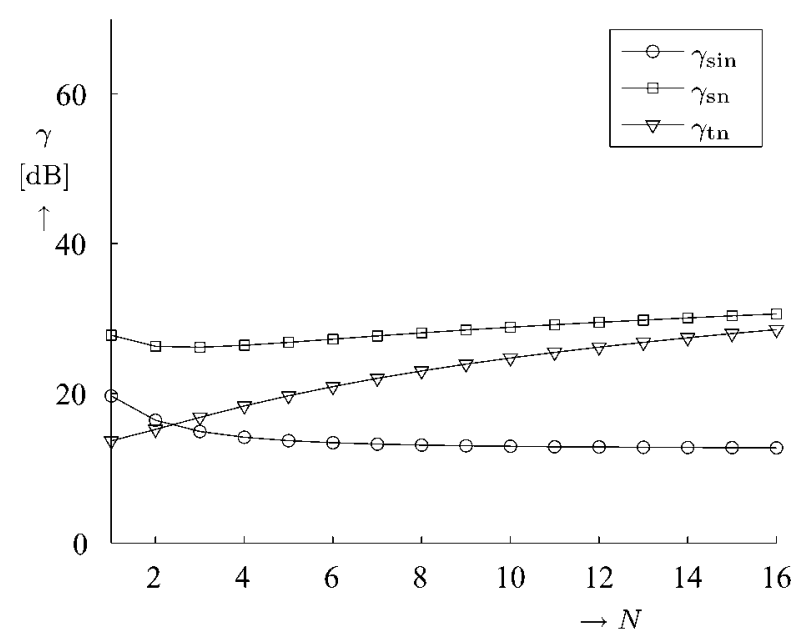

(b)

Fig. 5. (a) Maximum bit rate per channel $R_{\mathrm{b}, \max }$ as a function of number of simultaneously active channels $N$ in a PA with 16 transmitters and receivers and BPSK modulation. (b) Corresponding SNRs.

at $1 \mathrm{~Gb} / \mathrm{s}$. Obviously, shot noise and thermal noise hardly play a role in this numerical example; the maximum bit rate is mainly limited by the source-induced noise. This also explains why the maximum bit rate decreases so fast with increasing number of channels: the number of OBI noise terms is proportional to the square of the number of channels; so, in this case, the maximum bit rate is roughly inversely proportional to the square of the number of channels.

Therefore, in an application with a fixed number of transmitters, it makes sense to turn off the optical sources in the transmitters that are inactive, so that the number of OBI noise terms decreases, and the bit rates of the remaining transmitters can, hence, be increased. This is illustrated in Fig. 5(a), where the maximum bit rate per channel $R_{\mathrm{b}}$ that can be achieved at a BER of $10^{-9}$ has been plotted as a function of the number of simultaneously active channels in a PA with a fixed number of 16 transmitters and receivers. The corresponding SNRs are shown in Fig. 5(b). All of these were calculated using the same formulas as in the earlier example, with the difference that the network

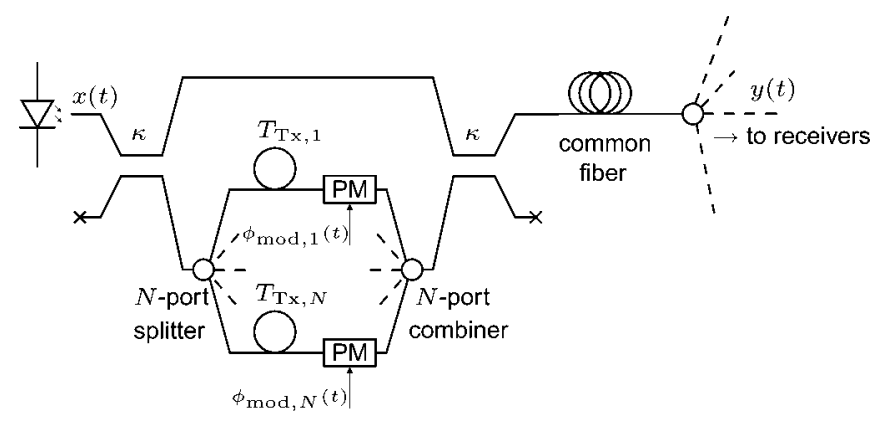

Fig. 6. SIRL system.

losses now have a fixed value $L_{\mathrm{nw}} \approx 1.05 \times 16^{2} \approx 268$ rather than $1.05 \mathrm{~N}^{2}$.

From Fig. 5(a), it follows that at most 2 channels can be active at $10 \mathrm{~Gb} / \mathrm{s}$, and at most 11 channels can be active at $1 \mathrm{~Gb} / \mathrm{s}$. Again, shot noise hardly plays a role in this numerical example. For large values of $N$, the performance is limited by OBI noise, whereas for low values of $N$, the performance is limited by thermal noise. Note that, for $N=16$, the values in Fig. 5 are the same as in Fig. 4, whereas for lower values of $N$, the maximum bit rates in Fig. 5(a) are lower than the ones in Fig. 4(a), due to the larger network losses. The main conclusion, however, is that the bit rates of individual channels can be significantly increased when some other channels become inactive. Stated otherwise, in a CM network with a fixed bit rate, errors will tend to occur when too many users are simultaneously active. This is further considered in [16], where some protocols are proposed for controlling the traffic in a packet-switched PA-based CM system.

\section{SingLE INTRINSIC REFERENCE LAdDER SyStem}

\section{A. System Description}

The IRL system that was proposed by Brooks et al [3] is actually similar to the PA. The only difference is that the IRL has a common source; its power is distributed over the coherence modulators using splitters. The input signals of the different coherence modulators can be made mutually incoherent by inserting suitable delays. However, this will always result in a lower transmitted power than in case of the PA, whereas the OBI noise performance will be approximately the same.

However, the OBI noise performance can be improved by letting all the channels share the same reference light wave. This can be achieved by means of the optical circuit in Fig. 6, which is termed the SIRL system. It uses a common transmission unit, which is basically an MZI with $N$ subpaths in the lower path, each having its own PDD $T_{\mathrm{Tx}, i}$ with the upper (reference) path, and phase modulator with modulating signal $\phi_{\bmod , i}(t)$. The ratio between the power in the reference light wave and the modulated light waves is controlled by means of the coupling constant $\kappa$ of the directional couplers. The splitter and combiner in the lower path have intrinsic splitting/combining loss $N$, and their excess loss is assumed to be incorporated in the excess loss $L_{\mathrm{Tx}}$ of the whole transmission unit and the coupling constant $\kappa$. The remaining part of the system is assumed to have the same characteristics as the PA described in Section II-A. 


\section{B. Multiplexed Signal}

The signal that is received by all coherence demodulators can, hence, be written as

$$
\begin{aligned}
y(t)= & \frac{1}{\sqrt{L_{\mathrm{Tx}} L_{\mathrm{nw}}}} \\
& \times\left[(1-\kappa) x(t)-\frac{\kappa}{N} \sum_{i=1}^{N} x\left(t-T_{\mathrm{Tx}, i}\right) \exp \left(j \phi_{\mathrm{mod}, i}(t)\right)\right]
\end{aligned}
$$

with autocorrelation function

$$
\begin{aligned}
& R_{y^{*} y}\left(t_{1}, t_{2}\right) \approx \frac{1}{L_{\mathrm{Tx}} L_{\mathrm{nw}}}\left\{(1-\kappa)^{2} R_{x^{*} x}\left(t_{2}-t_{1}\right)\right. \\
& -\frac{\kappa(1-\kappa)}{N} \sum_{i=1}^{N}\left[R_{x^{*} x}\left(t_{2}-t_{1}-T_{\mathrm{Tx}, i}\right) \exp \left(j \phi_{\mathrm{mod}, i}\left(t_{1}\right)\right)\right. \\
& \left.\quad+R_{x^{*} x}\left(t_{2}-t_{1}+T_{\mathrm{Tx}, i}\right) \exp \left(-j \phi_{\bmod , i}\left(t_{1}\right)\right)\right] \\
& +\frac{\kappa^{2}}{N^{2}} \sum_{i_{1}=1}^{N} \sum_{i_{2}=1}^{N} R_{x^{*} x}\left(t_{2}-t_{1}+T_{\mathrm{Tx}, i_{1}}-T_{\mathrm{Tx}, i_{2}}\right) \\
& \left.\quad \times \exp \left(j \phi_{\bmod , i_{2}}\left(t_{1}\right)-j \phi_{\bmod , i_{1}}\left(t_{1}\right)\right)\right\}
\end{aligned}
$$

\section{Receiver Output Signal}

Substituting (22) into (5) and taking into account that $R_{x^{*} x}(\tau)$ is negligible for $|\tau| \gg \tau_{\mathrm{c}}$, it follows that the expected value of the output signal of receiver $r$ can be written as

$$
\begin{aligned}
& E\left[V_{\mathrm{LP}, r}(t)\right] \approx \frac{Z_{\mathrm{TIA}} R_{\mathrm{pd}}}{2 L_{\mathrm{Tx}} L_{\mathrm{nw}} L_{\mathrm{Rx}}} \int h_{\mathrm{LP}}(t-\rho) \\
& \times\left[\frac{\kappa(1-\kappa)}{N} \sum_{i=1}^{N} \operatorname{Re}\left\{R_{x^{*} x}\left(T_{\mathrm{Rx}, r}-T_{\mathrm{Tx}, i}\right) \exp \left(j \phi_{\mathrm{mod}, i}(\rho)\right)\right\}\right. \\
& -\frac{\kappa^{2}}{N^{2}} \sum_{i_{1}=1}^{N} \sum_{i_{2}=1}^{N} \operatorname{Re}\left\{R_{x^{*} x}\left(T_{\mathrm{Rx}, r}+T_{\mathrm{Tx}, i_{1}}-T_{\mathrm{Tx}, i_{2}}\right)\right. \\
& \left.\left.\quad \times \exp \left(j \phi_{\mathrm{mod}, i_{2}}(\rho)-j \phi_{\mathrm{mod}, i_{1}}(\rho)\right)\right\}\right] \mathrm{d} \rho
\end{aligned}
$$

It follows that the output signal of each receiver $r$ depends only on the modulating signal of the corresponding transmitter $r$ if $T_{\mathrm{Rx}, r}=T_{\mathrm{Tx}, r},\left|T_{\mathrm{Rx}, r}-T_{\mathrm{Tx}, i}\right| \gg \tau_{\mathrm{c}}$ for $i \neq r$, and $\left|T_{\mathrm{Rx}, r}+T_{\mathrm{Tx}, i_{1}}-T_{\mathrm{Tx}, i_{2}}\right| \gg \tau_{\mathrm{c}}$ for any $i_{1}, i_{2}$. The resulting output signal at the sampling instants in case of BPSK modulation and matched filtering (see Section II-E) is then

$$
E\left[V_{\mathrm{LP}, r}(t)\right] \approx \pm \frac{\kappa(1-\kappa) Z_{\mathrm{TIA}} R_{\mathrm{pd}} P_{\mathrm{in}} T_{\mathrm{s}}}{N L_{\mathrm{Tx}} L_{\mathrm{Rx}} L_{\mathrm{nw}}} .
$$

\section{Choice of the Path Delay Differences}

From the conditions in the previous section, it follows that the PDDs in the SIRL transmitter should satisfy the same conditions as in the PA (individual PDDs and their mutual dif- ferences should be much larger than $\tau_{\mathrm{c}}$ ) and that the difference between any individual PDD and any mutual difference $\left(T_{\mathrm{Tx}, i_{1}}-\left|T_{\mathrm{Tx}, i_{2}}-T_{\mathrm{Tx}, i_{3}}\right|\right)$ should be much larger than $\tau_{\mathrm{c}}$.

A straightforward choice is to choose some delay value $T$ with $T \gg \tau_{\mathrm{c}}$ and then set the PDDs as odd multiples of $T$, so that $T_{\mathrm{Tx}, i}=(2 i-1) T_{\mathrm{Tx}, 1}$. Or alternatively, one could choose $T_{\mathrm{Tx}, i}=(N+i-1) T$. In both cases, the largest PDD would be $(2 N-1) T$.

Now, consider a similar numerical example as in Section II-D. Using (1), (5), and (22), it can be verified that the theoretical crosstalk can still be kept well below $-90 \mathrm{~dB}$ by choosing $T \geq 3 \tau_{\mathrm{c}}$. The resulting maximum PLD will then be $(2 \times 16-1) \times 60 \mu \mathrm{m} \approx 1.9 \mathrm{~mm}$, which is still well realizable.

\section{E. Noise Analysis}

The noise variance at the output of the low-pass filter can be calculated in a similar way as in Section II-E. Using (21), we find

$$
\begin{aligned}
& R_{y^{*} y}\left(\rho_{1}, \rho_{2}\right) R_{y^{*} y}\left(\rho_{2}-T_{\mathrm{Rx}, r}, \rho_{1}-T_{\mathrm{Rx}, r}\right)=\frac{1}{L_{\mathrm{Tx}}^{2} L_{\mathrm{nw}}^{2}} \\
& \times\left\{\left[(1-\kappa)^{4}+\frac{2(1-\kappa)^{2} \kappa^{2}}{N}+\frac{\kappa^{4}}{N^{2}}\right]\left|R_{x^{*} x}\left(\rho_{2}-\rho_{1}\right)\right|^{2}\right. \\
& +\frac{\kappa^{2}(1-\kappa)^{2}}{N^{2}} \sum_{i=1}^{N}\left[\left|R_{x^{*} x}\left(\rho_{2}-\rho_{1}-T_{\mathrm{Tx}, i}\right)\right|^{2}\right. \\
& \left.+\left|R_{x^{*} x}\left(\rho_{2}-\rho_{1}+T_{\mathrm{Tx}, i}\right)\right|^{2}\right] \\
& \left.+\frac{\kappa^{4}}{N^{4}} \sum_{i_{1}=1}^{N} \sum_{\substack{i_{2}=1 \\
i_{2} \neq i_{1}}}^{N}\left|R_{x^{*} x}\left(\rho_{2}-\rho_{1}+T_{\mathrm{Tx}, i_{1}}-T_{\mathrm{Tx}, i_{2}}\right)\right|^{2}\right\} \\
& R_{y^{*} y}\left(\rho_{1}-T_{\mathrm{Rx}, r}, \rho_{2}\right) R_{y^{*} y}\left(\rho_{2}-T_{\mathrm{Rx}, r}, \rho_{1}\right) \\
& =\frac{\kappa^{2}(1-\kappa)^{2}}{N^{2} L_{\mathrm{Tx}}^{2} L_{\mathrm{nw}}^{2}}\left|R_{x^{*} x}\left(\rho_{2}-\rho_{1}\right)\right|^{2} \exp \left(j 2 \phi_{\bmod , r}\left(\rho_{1}\right)\right) \\
& R_{y^{*} y}(\rho, \rho)=R_{y^{*} y}\left(\rho-T_{\mathrm{Rx}, r}, \rho-T_{\mathrm{Rx}, r}\right) \\
& =\frac{2 P_{\mathrm{in}}}{L_{\mathrm{Tx}} L_{\mathrm{nw}}}\left[(1-\kappa)^{2}+\frac{\kappa^{2}}{N}\right] \text {. }
\end{aligned}
$$

Substituting these in (8) and assuming BPSK modulation and matched filtering results in

$$
\begin{aligned}
\sigma_{V_{\mathrm{LP}, r}(t)}^{2} \approx & Z_{\mathrm{TIA}}^{2} T_{\mathrm{s}}\left\{\frac { R _ { \mathrm { pd } } ^ { 2 } P _ { \mathrm { in } } ^ { 2 } \tau _ { \mathrm { c } } } { 2 L _ { \mathrm { Tx } } ^ { 2 } L _ { \mathrm { Rx } } ^ { 2 } L _ { \mathrm { nw } } ^ { 2 } } \left[(1-\kappa)^{4}\right.\right. \\
& \left.+\frac{(4 N+1)(1-\kappa)^{2} \kappa^{2}}{N^{2}}+\frac{(2 N-1) \kappa^{4}}{N^{3}}\right] \\
& \left.+\frac{R_{\mathrm{pd}} P_{\mathrm{in}} e}{L_{\mathrm{Tx}} L_{\mathrm{Rx}} L_{\mathrm{nw}}}\left[(1-\kappa)^{2}+\frac{\kappa^{2}}{N}\right]+S_{\mathrm{th}}\right\} .
\end{aligned}
$$


Using (23) and (27), the SNR can be written as in (14), with

$$
\begin{aligned}
\gamma_{\mathrm{sin}}= & N \kappa^{2}(1-\kappa)^{2} T_{\mathrm{s}} \tau_{\mathrm{c}}^{-1}\left[N^{3}(1-\kappa)^{4}\right. \\
& \left.+\left(4 N^{2}+N\right)(1-\kappa)^{2} \kappa^{2}+(2 N-1) \kappa^{4}\right]^{-1} \\
\gamma_{\mathrm{sn}}= & \frac{\kappa^{2}(1-\kappa)^{2} R_{\mathrm{pd}} P_{\mathrm{in}} T_{\mathrm{s}}}{2 N\left[N(1-\kappa)^{2}+\kappa^{2}\right] L_{\mathrm{Tx}} L_{\mathrm{Rx}} L_{\mathrm{nw}} e} \\
\gamma_{\mathrm{tn}}= & \frac{\kappa^{2}(1-\kappa)^{2} R_{\mathrm{pd}}^{2} P_{\mathrm{in}}^{2} T_{\mathrm{s}}}{2 N^{2} L_{\mathrm{Tx}}^{2} L_{\mathrm{Rx}}^{2} L_{\mathrm{nw}}^{2} S_{\mathrm{th}}} .
\end{aligned}
$$

The BER still follows from (18). Obviously, the performance of the SIRL system depends on the coupling coefficient $\kappa$, which determines the ratio between the reference light wave and the modulated light waves. The performance is optimized by maximizing the expression for the SNR $\gamma$ with respect to $\kappa$. In general, this cannot be done analytically.

\section{F. Numerical Example}

Consider a numerical example of an SIRL system for $N$ CM channels, with the same parameter values as in Section II-F. The only difference is caused by the fact that no combiner is required, since the $\mathrm{CM}$ channels are generated in a common transmission unit. The network losses, therefore, become $L_{\mathrm{nw}} \approx 1.02 N$.

The maximum bit rate per channel $R_{\mathrm{b}}$ that can be achieved at a BER of $10^{-9}$ has been plotted as a function of the number of channels $N$ in Fig. 7(a), where, for each value of $N, R_{\mathrm{b}, \max }$ is numerically optimized with respect to the coupling coefficient $\kappa$. In the same figure, the maximum bit rates are plotted for the case $\kappa=1 / 2$. In Fig. 7(b), the optimum value of $\kappa$ has been plotted as a function of the number of channels. Obviously, the maximum bit rates that can be achieved with this SIRL system are significantly enhanced by setting $\kappa$ to its optimum value $\kappa_{\text {opt }}$ rather than simply choosing $\kappa=1 / 2$, especially for large values of $N$. The value of $\kappa_{\mathrm{opt}}$ increases when the number of channels $N$ increases. At $10 \mathrm{~Gb} / \mathrm{s}$, at most five channels can be supported when $\kappa=1 / 2$, and at most seven channels can be supported when $\kappa$ is optimized.

In Fig. 7(c), the corresponding SNRs are plotted as a function of $N$ for $\kappa=\kappa_{\mathrm{opt}}$. Obviously, the performance of the SIRL system is still mainly limited by source-induced noise, like the PA in Section II-F. The great advantage of the SIRL system with respect to the PA, however, is that the signal-to-sourceinduced-noise ratio of the SIRL system decreases much more slowly with increasing number of channels $N$ than for the PA. Maximizing (28) with respect to $\kappa$ results in an expression that decreases roughly inversely proportional to $N$, whereas (15) decreases roughly inversely proportional to $N^{2}$. This explains why the maximum bit rates in Fig. 7(a) are so much higher than the ones in Fig. 4(a), especially for large values of $N(3.4 \mathrm{~Gb} / \mathrm{s}$ for the SIRL system versus $0.5 \mathrm{~Gb} / \mathrm{s}$ for the PA when $N=16$ ).

A disadvantage of the SIRL system that stems from employing one common source is that the amount of OBI noise cannot be reduced when some channels become inactive, as was explained for the PA in Fig. 5. Hence, for an SIRL with a fixed number of channels (with orthogonal codes), the maximum bit rate per channel does not depend on the number of channels that are actually active.

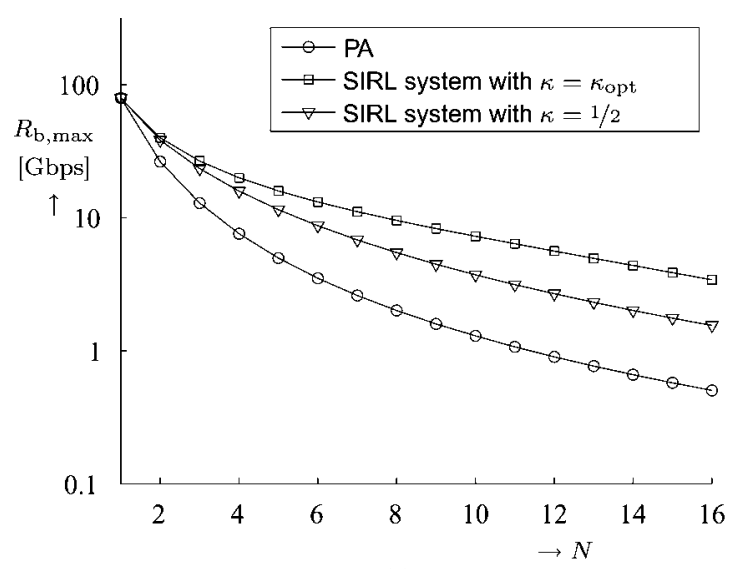

(a)

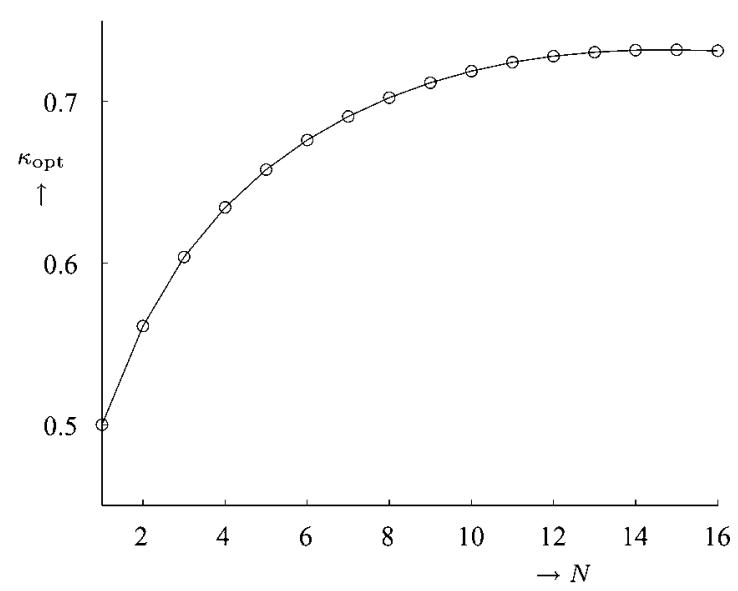

(b)

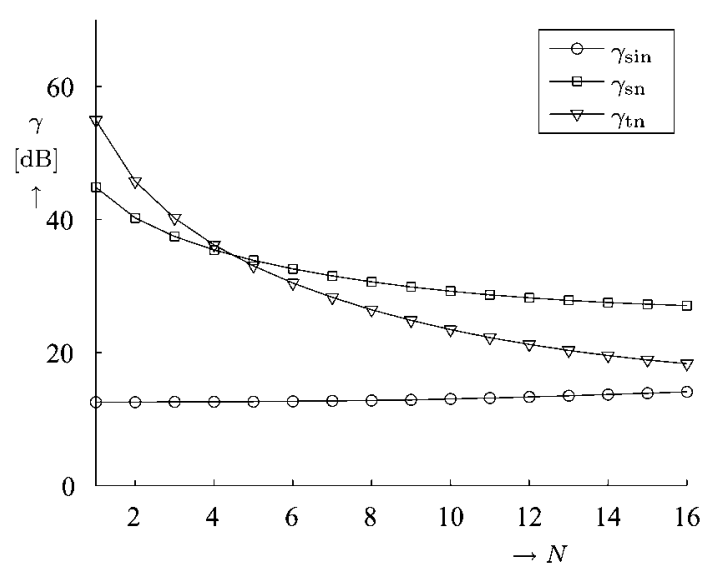

(c)

Fig. 7. Maximum bit rate per channel $R_{\mathrm{b}}$ and corresponding SNRs as a function of the number of channels $N$ in an SIRL system with BPSK modulation. For comparison, the maximum bit rates for the PA [Fig. 4(a)] are also plotted. (a) $R_{\mathrm{b}, \max }$. (b) Optimized coupling coefficient $\kappa$. (c) SNRs.

\section{DisContinuous SERIES SySTEM}

\section{A. System Description}

The DS system also has a common source, and is constructed by serially cascading two-arm coherence modulators, as illustrated in Fig. 8. Each coherence modulator has its own PDD 


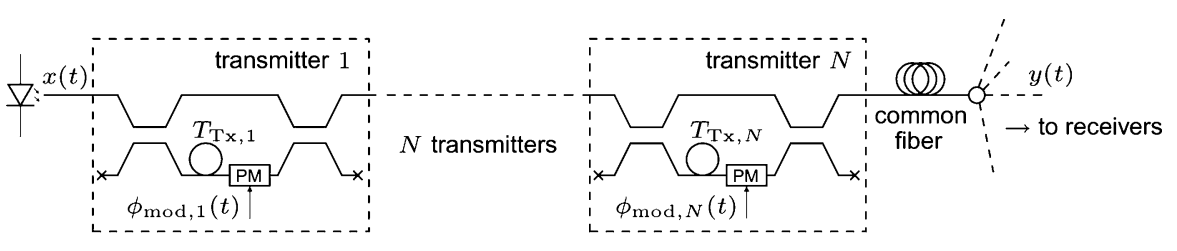

Fig. 8. DS system.

$T_{\mathrm{Tx}, i}$, phase modulator with modulating signal $\phi_{\bmod , i}(t)$, and excess loss $L_{\mathrm{Tx}}$, just like the PA in Section II.

\section{B. Multiplexed Signal}

The number of terms in the optical signal is doubled each time it goes through a coherence modulator. The signal that is received by all coherence demodulators can be written as

$$
\begin{aligned}
y(t)= & \frac{1}{2^{N} \sqrt{L_{\mathrm{Tx}}^{N} L_{\mathrm{nw}}}} \sum_{k_{1}=0}^{1} \cdots \sum_{k_{N}=0}^{1} x\left(t-\sum_{i=1}^{N} k_{i} T_{\mathrm{Tx}, i}\right) \\
& \times \exp \left\{j k_{1}\left[\pi+\phi_{\bmod , 1}\left(t-\sum_{i=2}^{N} k_{i} T_{\mathrm{Tx}, i}\right)\right]\right. \\
& +j k_{2}\left[\pi+\phi_{\bmod , 2}\left(t-\sum_{i=3}^{N} k_{i} T_{\mathrm{Tx}, i}\right)\right] \\
& \left.+\cdots+j k_{N}\left[\pi+\phi_{\bmod , N}(t)\right]\right\}
\end{aligned}
$$

with autocorrelation function

$$
\begin{aligned}
& R_{y^{*} y}\left(t_{1}, t_{2}\right)= \frac{1}{4^{N} L_{\mathrm{Tx}}^{N} L_{\mathrm{nw}}} \sum_{k_{1}=0}^{1} \ldots \sum_{k_{N}=0}^{1} \\
& \times \sum_{l_{1}=0}^{1} \cdots \sum_{l_{N}=0}^{1} R_{x^{*} x}\left(t_{2}-t_{1}+\sum_{i=1}^{N}\left(k_{i}-l_{i}\right) T_{\mathrm{Tx}, i}\right) \\
& \times \exp \left\{-j k_{1}\left[\pi+\phi_{\bmod , 1}\left(t_{1}-\sum_{i=2}^{N} k_{i} T_{\mathrm{Tx}, i}\right)\right]\right. \\
&+j l_{1}\left[\pi+\phi_{\bmod , 1}\left(t_{2}-\sum_{i=2}^{N} l_{i} T_{\mathrm{Tx}, i}\right)\right] \\
&-j k_{2}\left[\pi+\phi_{\bmod , 2}\left(t_{1}-\sum_{i=3}^{N} k_{i} T_{\mathrm{Tx}, i}\right)\right] \\
&+j l_{2}\left[\pi+\phi_{\bmod , 2}\left(t_{2}-\sum_{i=3}^{N} l_{i} T_{\mathrm{Tx}, i}\right)\right] \\
&+\cdots-j k_{N}\left[\pi+\phi_{\bmod , N}\left(t_{1}\right)\right] \\
&\left.-j l_{N}\left[\pi+\phi_{\bmod , N}\left(t_{2}\right)\right]\right\} .
\end{aligned}
$$

\section{Receiver Output Signal}

Substituting (32) into (5) and taking into account that $R_{x^{*} x}(\tau)$ is negligible for $|\tau| \gg \tau_{\mathrm{c}}$, it follows that the expected value of the output signal of receiver $r$ can be written as

$$
\begin{aligned}
& E\left[V_{\mathrm{LP}, r}(t)\right] \approx-\frac{Z_{\mathrm{TIA}} R_{\mathrm{pd}}}{2 \times 4^{N} L_{\mathrm{Tx}}^{N} L_{\mathrm{Rx}} L_{\mathrm{nw}}} \\
& \times \sum_{k_{1}=0}^{1} \cdots \sum_{k_{N}=0}^{1} \sum_{l_{1}=0}^{1} \cdots \sum_{l_{N}=0}^{1} \int h_{\mathrm{LP}}(t-\rho) \\
& \times \operatorname{Re}\left\{R_{x^{*} x}\left(T_{\mathrm{Rx}, r}+\sum_{i=1}^{N}\left(k_{i}-l_{i}\right) T_{\mathrm{Tx}, i}\right)\right. \\
& \times \exp \left[j\left(k_{1}-l_{1}+k_{2}-l_{2}+\cdots+k_{N}-l_{N}\right) \pi\right. \\
& +j k_{1} \phi_{\bmod , 1}\left(\rho-T_{\mathrm{Rx}, r}-\sum_{i=2}^{N} k_{i} T_{\mathrm{Tx}, i}\right) \\
& -j l_{1} \phi_{\bmod , 1}\left(\rho-\sum_{i=2}^{N} l_{i} T_{\mathrm{Tx}, i}\right) \\
& +j k_{2} \phi_{\bmod , 2}\left(\rho-T_{\mathrm{Rx}, r}-\sum_{i=3}^{N} k_{i} T_{\mathrm{Tx}, i}\right) \\
& -j l_{2} \phi_{\bmod , 2}\left(\rho-\sum_{i=3}^{N} l_{i} T_{\mathrm{Tx}, i}\right)+\cdots \\
& \left.\left.+j k_{N} \phi_{\bmod , N}\left(\rho-T_{\mathrm{Rx}, r}\right)-j l_{N} \phi_{\bmod , N}(\rho)\right]\right\} \mathrm{d} \rho \text {. }
\end{aligned}
$$

Now, the PDDs need to be chosen such that for each value of $r$, $\phi_{\text {mod, } r}(t)$ is the only modulating signal that becomes observable in this output signal. For the DS system, this is actually a far more complicated problem than for the PA and SIRL system. By carefully inspecting (33), one can state that the following two conditions have to be satisfied.

1) The relations between the PDDs should be chosen such that the value of $\left|T_{\mathrm{Rx}, r}+\sum_{i=1}^{N}\left(k_{i}-l_{i}\right) T_{\mathrm{Tx}, i}\right|$ is:

a) much smaller than $\tau_{\mathrm{c}}$ if and only if $k_{i}=l_{i}$ for all $i \neq r, k_{r}=0$, and $l_{r}=1$ (this can simply be done by setting $T_{\mathrm{Rx}, r}=T_{\mathrm{Tx}, r}$;

b) much larger than $\tau_{\mathrm{c}}$ in all other cases. 
2) The values of the PDDs $T_{\mathrm{Rx}, r}$ should be much smaller than the inverse bandwidths of the modulating signals, such that, for example, the last two terms in the cosine in (33) indeed cancel each other when $k_{N}=l_{N}=1$.

If the first condition is not satisfied, crosstalk will occur in a similar way as described for the PA and SIRL system. If the second condition is not satisfied, crosstalk will occur due to the fact that terms that are supposed to cancel each other are actually shifted in time with respect to each other.

When the conditions are satisfied (we will come back to this in the next section), however, we can write (33) as

$$
\begin{aligned}
E\left[V_{\mathrm{LP}, r}(t)\right] \approx & \frac{Z_{\mathrm{TIA}} R_{\mathrm{pd}} P_{\mathrm{in}}}{4^{N} L_{\mathrm{Tx}}^{N} L_{\mathrm{Rx}} L_{\mathrm{nw}}} \int h_{\mathrm{LP}}(t-\rho) \\
& \times \sum_{k_{1}=0}^{1} \cdots \sum_{k_{r}-1=0}^{1} \sum_{k_{r+1}=0}^{1} \cdots \sum_{k_{N}=0}^{1} \\
& \times \cos \left[\phi_{\mathrm{mod}, r}\left(\rho-\sum_{i=r+1}^{N} k_{i} T_{\mathrm{Tx}, i}\right)\right] \mathrm{d} \rho .
\end{aligned}
$$

This actually reveals a disadvantage of the DS system: the output signal does not simply contain just one term-like in the PA and SIRL system-but rather $2^{N-1}$ terms, which can be reduced to $2^{N-r}$ terms that are time-shifted with respect to each other. This generally results in a distortion of the output signal. The most severe distortion occurs in receiver 1: the maximum delay difference between the terms is then $\sum_{i=2}^{N} T_{\mathrm{Tx}, i}$. Hence, the distortion can only be neglected when this value is much smaller than the inverse of the bandwidth of the modulating signals. (Note that this implies that the largest PDD value should actually be assigned to $T_{\mathrm{Tx}, 1}$.) The expected output signal at the sampling instants in case of BPSK modulation and matched filtering (see Section II-E), with symbol time $T_{\mathrm{s}} \gg \sum_{i=2}^{N} T_{\mathrm{Tx}, i}$, can, hence, be simplified to

$$
E\left[V_{\mathrm{LP}, r}(t)\right] \approx \pm \frac{Z_{\mathrm{TIA}} R_{\mathrm{pd}} P_{\mathrm{in}} T_{\mathrm{s}}}{2^{N+1} L_{\mathrm{Tx}}^{N} L_{\mathrm{Rx}} L_{\mathrm{nw}}} .
$$

\section{Choice of the PDDs}

As already mentioned in the previous section, the problem of choosing the suitable PDD values for the DS system is far from straightforward. Suppose we again do this by choosing some delay value $T \gg \tau_{\mathrm{c}}$, and choose the PDDs as suitable integer multiples of $T_{\mathrm{Tx}, 1}$, in such a way that the conditions mentioned in the previous section are met. This is a rather involved mathematical problem, which has been studied by Bløtekjær et al. [21]. They designed several procedures for choosing the PDDs based on different criteria, such as minimizing the value of the largest PDD, minimizing the sum of all the PDDs, and minimizing the ratio between minimum and maximum PDD. Here, we will consider the solution for the minimum sum of the PDDs, since this will actually result in minimum distortion. This prescribes the PDDs to be chosen according to the recursive equation [21]

$$
T_{\mathrm{Tx}, i}=T_{\mathrm{Tx}, i-1}+T+\sum_{k=1}^{i-1} T_{\mathrm{Tx}, k} \text { with } T_{\mathrm{Tx}, 1}=T
$$

or in closed form

$$
T_{\mathrm{Tx}, i}=\frac{1}{\sqrt{5}}\left[\left(\frac{3+\sqrt{5}}{2}\right)^{i}-\left(\frac{3-\sqrt{5}}{2}\right)^{i}\right] T .
$$

An advantage of this solution with respect to the other solutions that were proposed in [21] is that the individual PDDs do not depend on the total number of channels $N$, so that an existing DS system with minimal sum of the PDDs can be extended by one coherence modulator without changing the values of the present coherence modulators, while still keeping the sum of the PDDs minimal. Obviously, the resulting PDD values increase very rapidly (exponentially) with increasing number of channels $N$. This is also the case for the other solutions presented in [21].

Now, consider a similar numerical example as in Section II-D and III-D. If we again choose $T=3 \tau_{\mathrm{c}}$, the resulting maximum PLD will be $2,178,309 \times 60 \mu \mathrm{m} \approx 131 \mathrm{~m}$. Obviously, this is not the practical value for realization in an optical integrated circuit. If the PLD is to be kept below a value of, say, $1 \mathrm{~cm}$, then it can be verified that at most 6 (instead of 16) channels can be supported by the DS system. If the MZI with the largest PDD $\left(T_{\mathrm{Tx}, 6}\right)$ is put in front, then the maximum delay between the terms in the received signal is $\sum_{i=1}^{5} T_{\mathrm{Tx}, i}=88 \times 3 \tau_{\mathrm{c}}=26.4$ ps, so distortion can be neglected for bit rates well below $38 \mathrm{~Gb} / \mathrm{s}$.

\section{E. Noise Analysis}

For convenience, it will be assumed that only a few channels are supported, so that the maximum delay spread can be kept much smaller than the inverse bandwidth of the modulating signals. Therefore, we can simplify (32) to

$$
\begin{aligned}
& R_{y^{*} y}\left(t_{1}, t_{2}\right)=\frac{1}{4^{N} L_{\mathrm{Tx}}^{N} L_{\mathrm{nw}}} \sum_{k_{1}=0}^{1} \cdots \sum_{k_{N}=0}^{1} \\
& \quad \times \sum_{l_{1}=0}^{1} \cdots \sum_{l_{N}=0}^{1} R_{x^{*} x}\left(t_{2}-t_{1}+\sum_{i=1}^{N}\left(k_{i}-l_{i}\right) T_{\mathrm{Tx}, i}\right) \\
& \quad \times \exp \left\{-j \sum_{i=1}^{N}\left(k_{i}-l_{i}\right)\left(\pi+\phi_{\bmod , i}\left(t_{1}\right)\right)\right\}
\end{aligned}
$$

so that we can write

$$
\begin{aligned}
& R_{y^{*} y}\left(\rho_{1}, \rho_{2}\right) R_{y^{*} y}\left(\rho_{2}-T_{\mathrm{Rx}, r}, \rho_{1}-T_{\mathrm{Rx}, r}\right) \\
& =\frac{1}{8^{N} L_{\mathrm{Tx}}^{2 N} L_{\mathrm{nw}}^{2}} \sum_{k_{1}=0}^{1} \cdots \sum_{k_{N}=0}^{1} \sum_{l_{1}=0}^{1} \cdots \sum_{l_{N}=0}^{1}\left[\prod_{i=1}^{N} f\left(k_{i}, l_{i}\right)\right] \\
& \quad \times\left|R_{x^{*} x}\left(\rho_{2}-\rho_{1}+\sum_{i=1}^{N}\left(k_{i}-l_{i}\right) T_{\mathrm{Tx}, i}\right)\right|^{2} \\
& R_{y^{*} y}\left(\rho_{1}-T_{\mathrm{Rx}, r}, \rho_{2}\right) R_{y^{*} y}\left(\rho_{2}-T_{\mathrm{Rx}, r}, \rho_{1}\right) \\
& \approx \frac{1}{4^{N+1} L_{\mathrm{Tx}}^{2 N} L_{\mathrm{nw}}^{2}}\left|R_{x^{*} x}\left(\rho_{2}-\rho_{1}\right)\right|^{2} \exp \left(j 2 \phi_{\bmod , r}\left(\rho_{1}\right)\right)
\end{aligned}
$$




$$
\begin{aligned}
& R_{y^{*} y}(\rho, \rho)=R_{y^{*} y}\left(\rho-T_{\mathrm{Rx}, r}, \rho-T_{\mathrm{Rx}, r}\right) \\
& \quad=\frac{2 P_{\mathrm{in}}}{2^{N} L_{\mathrm{Tx}}^{N} L_{\mathrm{nw}}}
\end{aligned}
$$

where

$$
f\left(k_{i}, l_{i}\right)= \begin{cases}1, & k_{i}=l_{i} \\ 1 / 2, & k_{i} \neq l_{i} .\end{cases}
$$

Substituting these in (8) and assuming BPSK modulation and matched filtering results in

$$
\begin{aligned}
\sigma_{V_{\mathrm{LP}, r}(t)}^{2} \approx & Z_{\mathrm{TIA}}^{2} T_{\mathrm{s}}\left\{\frac{\left[4(3 / 2)^{N}+1\right] R_{\mathrm{pd}}^{2} P_{\mathrm{in}}^{2} \tau_{\mathrm{c}}}{2^{2 N+3} L_{\mathrm{Tx}}^{2 N} L_{\mathrm{Rx}}^{2} L_{\mathrm{nw}}^{2}}\right. \\
& \left.+\frac{R_{\mathrm{pd}} P_{\mathrm{in}} e}{2^{N} L_{\mathrm{Tx}}^{N} L_{\mathrm{Rx}} L_{\mathrm{nw}}}+S_{\mathrm{th}}\right\} .
\end{aligned}
$$

Using (35) and (43), the SNR can be written as in (14), with

$$
\begin{aligned}
\gamma_{\mathrm{sin}} & =\frac{T_{\mathrm{s}}}{\left[4(3 / 2)^{N}+1\right] \tau_{\mathrm{c}}} \\
\gamma_{\mathrm{sn}} & =\frac{R_{\mathrm{pd}} P_{\mathrm{in}} T_{\mathrm{s}}}{2^{N+3} L_{\mathrm{Tx}}^{N} L_{\mathrm{Rx}} L_{\mathrm{nw}} e} \\
\gamma_{\mathrm{tn}} & =\frac{R_{\mathrm{pd}}^{2} P_{\mathrm{in}}^{2} T_{\mathrm{s}}}{2^{2 N+3} L_{\mathrm{Tx}}^{2 N} L_{\mathrm{Rx}}^{2} L_{\mathrm{nw}}^{2} S_{\mathrm{th}}} .
\end{aligned}
$$

The BER still follows from (18).

\section{F. Numerical Example}

Consider a numerical example of a DS system for $N$ CM channels, with the same parameter values as in Sections II-F and III-F. The network losses are the same as for the SIRL system: $L_{\mathrm{nw}} \approx 1.02 N$.

The maximum bit rate $R_{\mathrm{b}, \max }$ that can be achieved per channel at $P_{\mathrm{e}}=10^{-9}$ has been plotted as a function of the number of channels $N$ in Fig. 9(a), together with the results of the PA [Fig. 4(a)] and the ( $\kappa$-optimized) SIRL system [Fig. 7(a)]. The number of channels of the DS system has been limited to six, because of the restricted PDD values that can be fabricated (see Section IV-D). At most five channels can be supported at $10 \mathrm{~Gb} / \mathrm{s}$.

Note that these maximum bit rates have been derived assuming that the distortion due to delay spread in the output signal could be neglected. However, from (37), it follows that although the symbol times $T_{\mathrm{s}}$ corresponding to the bit rates in Fig. 9(a) are larger than the delay spreads $\sum_{i=1}^{N-1} T_{\mathrm{Tx}, i}$, the delay spread is not negligible. Hence, some intersymbol interference (ISI) will occur, so that the bit rates that can be achieved in practice are a bit smaller than the ones that are shown in Fig. 9(a). This will not be considered in further detail.

The corresponding SNRs are shown in Fig. 9(b). Obviously, the performance is mainly limited by source-induced noise for $N<6$; thermal noise becomes significant only when $N=6$.

An advantage of the DS system with respect to the PA and the SIRL system is that all received optical power is used for demodulation. This explains why the maximum bit rate decreases relatively slowly with increasing number of $\mathrm{CM}$ channels $N$ for

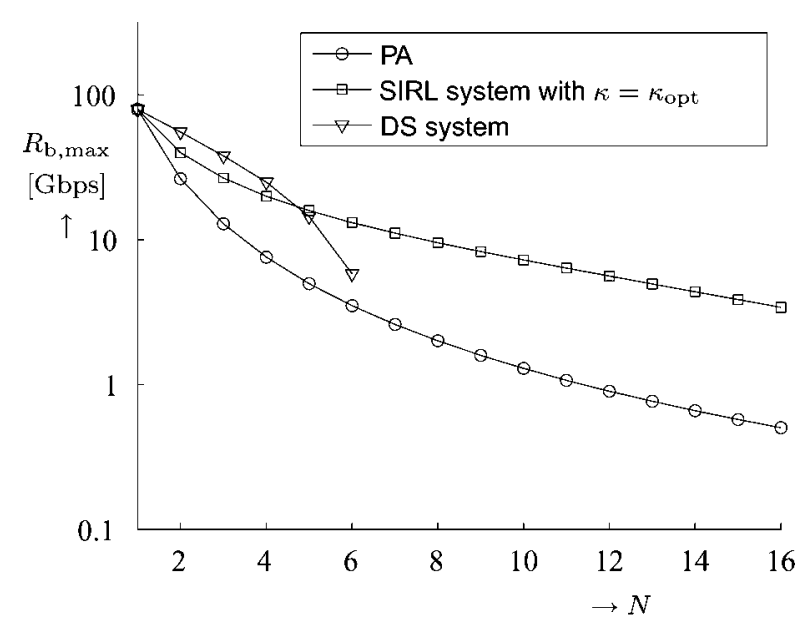

(a)

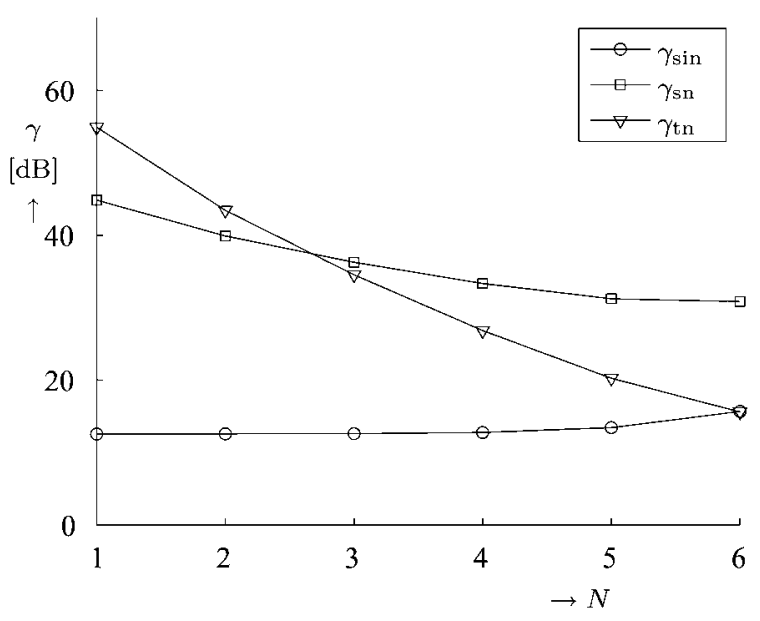

(b)

Fig. 9. (a) Maximum bit rate per channel $R_{\mathrm{b}, \max }$ as a function of the number of channels $N$ in a DS system with BPSK modulation. (b) Corresponding SNRs. For comparison, the maximum bit rates for the PA [Fig. 4(a)] and the ( $\kappa$-optimized) SIRL system [Fig. 7(a)] are also plotted.

low values of $N$. For higher values of $N$, however, the maximum bit rate decreases much faster, due to fact that both the number of OBI noise terms and the optical loss increase exponentially with $N$.

For a DS system with a fixed number of fixed coherence modulators (as shown in Fig. 8), the maximum bit rate per channel cannot be increased when some channels become inactive, because of the common source. However, this problem can be circumvented by equipping the MZIs in the coherence modulators with tunable couplers, so that inactive coherence modulators can direct all optical power through only on arm, which —apart from some excess loss-is more or less equivalent to completely removing the coherence modulator. Hence, for low excess loss, the maximum bit rates as a function of the number of simultaneously active channels are approximately the same as the ones plotted in Fig. 9(a).

\section{COMPARISON}

In the previous three sections, three different system topologies for performing CM have been explained, and compared 
with respect to design constraints and theoretical performance. In this section, they will be compared with respect to some practical aspects.

\section{A. Modulation Formats}

One particular difference between the topologies is the modulation formats that they can accommodate. Although only PM was considered in this paper, the PM in the single-channel system in Fig. 1 could be replaced by IM, provided that balanced detection is applied in the receiver. Digital transmission can then be performed using on-off keying (OOK) modulation [9]-[11], [14], [15]. This could be implemented by directly modulating the source, but this has the disadvantage that broadband optical sources generally have limited modulation bandwidths, in the order of a few hundred megahertz. External IM is, therefore, preferred. The advantage of IM compared to PM is that the modulator is taken out of the MZI, so that the modulation and channel definition functions are separated. This simplifies integration when these functions are realized in different technologies.

IM can also be applied in a PA, since it has completely independent optical channels. Application in the SIRL system is limited because it has a common optical source, so modulating the source signal would also modulate the common reference signal. IM could be implemented in an SIRL system by replacing the phase modulators by intensity modulators in the same position, but then IM looses its advantage with respect to PM. IM cannot be applied in a DS system, since the modulated signal from the first coherence modulator will also pass modulators in succeeding coherence modulators, resulting in a crosstalk between the CM channels.

\section{B. Flexibility}

A drawback of the SIRL system is that the transmitters need to be localized on a single optical chip, so that it can only be applied in network structures where the channels that are to be multiplexed are available in a single node. In a passive optical network (PON), for example, the SIRL system can only be used for the downstream channels and not for the upstream channels. The PA and DS system provide more flexibility in locating the transmitters, since they are realized as separate coherence modulators. The PA can be used in a star topology, and the DS system is restricted to a bus topology.

Adding channels is not equally difficult for each alternative. For the DS system, for example, adding a channel simply requires breaking the connection somewhere in the series connection of the coherence modulators, and then, inserting another coherence modulator. In case of the PA, coherence modulators and demodulators can be added as long as the splitters and combiners in the network provide enough ports. For the SIRL system, adding channels involves replacing the entire chip.

The PA and DS systems are also more flexible in the sense that channels can be easily switched off, hence reducing the amount of OBI noise in the receivers (and the network losses in case of the DS system), thereby creating an opportunity to increase the bit rates of the remaining channels, as discussed in Sections II-F and IV-F. Moreover, capacity could be allocated nonuniformly in the PA by increasing the transmitted powers of nodes that are supposed to transmit at higher rates than others. This is not further analyzed in detail.

\section{Complexity}

As far as circuit complexity is involved, the PA and DS system are obviously simpler than the SIRL system, although the realizability of the coherence modulators for the DS system can become an issue when a large number of channels is to be multiplexed, due to the PDD values that are required (see Section IV-D).

\section{Robustness}

From a cost point of view, it might be seen as an advantage that the SIRL system and DS system require only one common light source for all the transmitted channels, whereas the PA requires one light source for each channel. Note, however, that a light source-being an active device-is a typical component that might break down during operation. Hence, the single light source in the SIRL system and DS system can be considered as what is called a single point of failure. That is, a breakdown of the light source causes the entire network to be shut down, whereas in the PA, a failing light source only causes one channel to be extinguished. The robustness of the SIRL and DS system can be improved by inserting a backup light source at the (so far) unused lower input port of the left MZI in Figs. 6 and 8. The DS system is most vulnerable because any broken connection in the series connection of the coherence modulators will shut down the network.

\section{CONCLUSION}

Three CM topologies were explained, analyzed, and compared in this paper. Each topology turned out to have its advantages and disadvantages. Choice for a particular topology depends on several criteria, such as the required number of channels, the location of the transmission units, the frequencies and bandwidths of the signals to be transported, and the allowed system complexity. For a large number of channels, the SIRL system shows a superior noise performance compared to the PA and the DS system. However, the PA is a more universally applicable topology, since it provides more flexibility in locating the transmission units.

\section{ACKNOWLEDGMENT}

Dr. C. G. H. Roeloffzen is acknowledged for helpful discussions on integrated optics technology.

\section{REFERENCES}

[1] C. Delisle and P. Cielo, "Application de la modulation spectrale à la transmission de l'information (Application of spectral modulation to information transmission)," Can.J. Phys., vol. 53, pp. 1047-1053, 1975.

[2] P. Cielo and C. Delisle, "Multiplexage en communication optique par interférometrie à grande différence de marche en lumière blanche (Multiplexing in optical communications by interferometry with a large pathlength difference in white light)," Can.J. Phys., vol. 54, pp. 2322-2331, 1976 . 
[3] J. L. Brooks, R. H. Wentworth, R. C. Youngquist, M. Tur, B. Y. Kim, and H. J. Shaw, "Coherence multiplexing of fiber-optic interferometric sensors," J. Lightw. Technol., vol. LT-3, no. 5, pp. 1062-1072, Oct. 1985.

[4] J.-P. Goedgebuer, H. Porte, and A. Hamel, "Electrooptic modulation of multilongitudinal mode laser diodes: Demonstration at $850 \mathrm{~nm}$ with simultaneous data transmission by coherence multiplexing," IEEE J. Quantum Electron., vol. QE-23, no. 7, pp. 1135-1144, Jul. 1987.

[5] J.-P. Goedgebuer and A. Hamel, "Coherence multiplexing using a parallel array of electrooptic modulators and multimode semiconductor lasers," IEEE J. Quantum Electron., vol. QE-23, no. 12, pp. 2224-2237, Dec. 1987.

[6] R. H. Wentworth, "Theoretical noise performance of coherencemultiplexed interferometric sensors," J. Lightw. Technol., vol. 7, no. 6, pp. 941-956, Jun. 1989.

[7] K. W. Chu and F. M. Dickey, "Optical coherence multiplexing for interprocessor communications," Opt. Eng., vol. 30, no. 3, pp. 337-344, Mar. 1991.

[8] D. A. Blair and G. D. Cormack, "Optimal source linewidth in a coherence multiplexed optical fiber communications system," J. Lightw. Technol., vol. 10, no. 6, pp. 804-810, Jun. 1992.

[9] D. D. Sampson, G. J. Pendock, and R. A. Griffin, "Photonic code-division multiple-access communications," Fiber Integr. Opt., vol. 16, no. 2, pp. 129-157, 1997.

[10] G. J. Pendock and D. D. Sampson, "Capacity of coherence-multiplexed CDMA networks," Opt. Commun., vol. 143, pp. 109-117, 1997.

[11] J. Kani, K. Iwatsuki, N. Takachio, and N. Fujii, "A simple broad-band coherence multiplexed optical access network and its scalability," J. Lightw. Technol., vol. 19, no. 4, pp. 456-464, Apr. 2001.

[12] A. Meijerink, N. Niëns, G. H. L. M. Heideman, and W. van Etten, "Chromatic fiber dispersion in single-mode coherence multiplex systems," in Proc. 10th IEEE/CVT Symp. Benelux, Eindhoven, The Netherlands, Nov. 2003, p. 9.

[13] A. Meijerink, R. O. Taniman, G. H. L. M. Heideman, and W. van Etten, "Comparison of three coherence multiplex system topologies," in Proc. 8th IEEE/LEOS Symp. Benelux, Enschede, The Netherlands, Nov. 2003, pp. 141-144.

[14] A. Meijerink, G. H. L. M. Heideman, and W. van Etten, "Balanced optical phase diversity receivers for coherence multiplexing," J. Lightw. Technol., vol. 22, no. 11, pp. 2393-2408, Nov. 2004

[15] A. Meijerink. (2005, Oct.). Coherence multiplexing for optical communication systems. Ph.D. dissertation, Univ. Twente, Enschede, The Netherlands [Online]. Available: http://doc.utwente.nl/50841/

[16] R. J. Blokpoel, A. Meijerink, and W. van Etten, "Traffic control in coherence-multiplexed networks," in Proc. 12th IEEE/CVT Symp. Benelux, Enschede, The Netherlands, Nov. 2005, p. P5.

[17] A. Meijerink, R. O. Taniman, and W. van Etten, "Coherence-multiplexed optical RF feeder networks," J. Lightw. Technol., to be published.

[18] J. W. Goodman, Statistical Optics. New York: Wiley, 1985.

[19] R. A. Griffin, D. D. Sampson, and D. A. Jackson, "Demonstration of data-transmission using coherent correlation to reconstruct a coded pulse sequence," IEEE Photon. Technol. Lett., vol. 4, no. 5, pp. 513-515, May 1992.

[20] J. G. Proakis, Digital Communications, 4 ed. Singapore: McGraw-Hill, 1983.

[21] K. Bløtekjær, R. H. Wentworth, and H. J. Shaw, "Choosing relative optical path delays in series-topology interferometric sensor arrays," J. Lightw. Technol., vol. LT-5, no. 2, pp. 229-235, Feb. 1987.

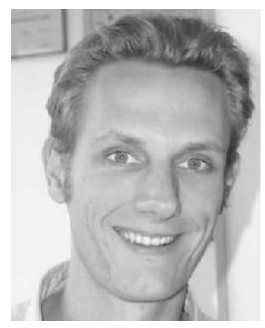

Arjan Meijerink (S'00-M'06) was born in Almelo, The Netherlands, in 1976. He received the M.Sc. and $\mathrm{Ph} . \mathrm{D}$. degrees (with honors) in electrical engineering from the University of Twente, Enschede, The Netherlands, in 2001 and 2005, respectively.

In 2000, he was with Ericsson Business Mobile Networks, Enschede, where he developed error concealment techniques for Bluetooth voice links. From 2001 to 2005, he was in the Telecommunication Engineering Group, University of Twente, where he is currently a Postdoctoral Researcher. His current research interests include optical communications and RF photonic signal processing techniques, particularly optical beam forming.

Dr. Meijerink received the Gauss Award for the Best Young Researcher's Presentation during the 22nd Symposium on Information and Communication Theory in the Benelux in May 2001 and the Second Prize in the IEEE Region 8 Student Paper Contest in July 2001.

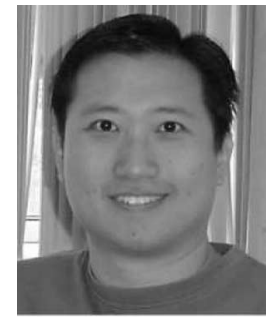

Robert O. Taniman ( $\mathrm{S}^{\prime} 06$ ) was born in Surabaya, Indonesia, in 1974. He received the B.Sc. degree (with honors) in engineering physics from the Institut Teknologi Sepuluh Nopember, Surabaya, in 1998, and the M.Sc. degree (with honors) in electrical engineering from the University of Twente, Enschede, The Netherlands, in 2003, where he is currently working toward the Ph.D. degree in the Chair for Design and Analysis of Communication Systems.

Between 1998 and 2001, he was an Assistant Lecturer at the University of Surabaya, Surabaya. His current research interests include the design and development of a resource allocation strategy for a multicarrier multimode passive optical network deployed as a broadband access network.

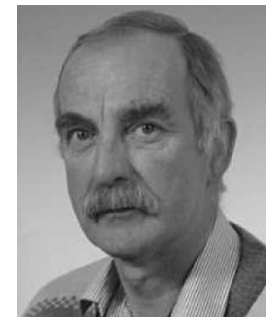

Geert H. L. M. Heideman was born in Oldenzaal, The Netherlands, in 1940. He received the M.Sc. degree in electrical engineering from Delft University of Technology, Delft, The Netherlands, in 1965.

In 1965, he was appointed Assistant Professor at the University of Twente, Enschede, The Netherlands, where, in 1974, he became a Senior Scientist, and in 1986, an Associate Professor. He was engaged in signal and information theory. He retired in 2003

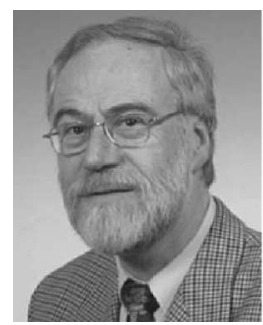

Wim van Etten (M'80-SM'91) was born in Zevenbergen, The Netherlands, in 1942. He received the M.Sc. and Ph.D. degrees in electrical engineering from Eindhoven University of Technology, Eindhoven, The Netherlands, in 1969 and 1976, respectively.

From 1969 to 1970, he was with Philips Electronics, where he developed circuits for oscilloscopes. In 1970, he became an Assistant Professor in the Faculty of Electrical Engineering, Eindhoven University of Technology, where, in 1985, he became an Associate Professor. From 1970 to 1976, he was engaged in research on the transmission of digital signals via coaxial and multiwire cables. Since 1976, he has been involved in research and education on optical fiber communications. In 1994, he became a full Professor of Telecommunications at the University of Twente, Enschede, The Netherlands. His current research interests include optical communications, mobile communications, detection, and simulation of communication systems. He is the author or coauthor of more than 80 papers in international journals, conferences, and symposia. He is the coauthor of the book Fundamentals of Optical Fiber Communications (Prentice Hall, 1991) and the author of the book Introduction to Random Signals and Noise (Wiley, 2005).

Prof. van Etten is a member of the Dutch Institute of Electronic and Radio Engineers (NERG) and the Chairman of the Executive Committee of the IEEE Benelux Joint Chapter on Communications and Vehicular Technology. He has also been a member of organizing committees and program committees of various conferences and symposia. Two of his papers were selected for a collection of 57 key papers on communications that appeared in the last 50 years in IEEE ComSoc publications. 